\title{
Prediction of preeclampsia developing at term
}

\author{
Iulia Huluta ${ }^{1}$, Anca Maria Panaitescu ${ }^{1,2}$ \\ 1 "Carol Davila" University of Medicine and Pharmacy, Bucharest, Romania \\ ${ }^{2}$ Filantropia Clinical Hospital, Bucharest, Romania
}

\begin{abstract}
Preterm preeclampsia (PE), occurring at $<37$ weeks' gestation, can be predicted from as early as 11-13 weeks and prevented with the use of aspirin. In contrast, term PE, which is more common than preterm-PE and it can be associated with important maternal morbidity and mortality, cannot be effectively predicted at 11-13 weeks and cannot be prevented by the prophylactic use of aspirin. This paper briefly reviews the pathogenesis of term PE and discusses strategies available for its prediction.
\end{abstract}

Key words: preeclampsia, aspirin, pravastatin, SFLT-1, PLGF

\section{INTRODUCTION}

Preeclampsia (PE) is one of the leading causes of maternal and fetal morbidity and mortality worldwide [1] and complicates about $3 \%$ of all pregnancies [2]. The pathogenesis of PE is not yet completely understood with some considering it a single disorder with a wide range of clinical manifestations [2-4] while others, as two distinct conditions [5].

Recent evidence suggests that preterm-PE, occurring at $<37$ weeks' gestation, is to a great extent predictable and preventable. Effective screening for preterm-PE can be provided by a combination of maternal factors, mean arterial pressure (MAP), Doppler assessment of the uterine artery pulsatility index (UTPI) and placental growth factor (PLGF) at 11-13 weeks' gestation with detection rate (DR) of $75 \%$ at false positive rate (FPR) of 10\% [2-4]. Prophylactic use of aspirin (aspirin $150 \mathrm{mg} /$ day from 11-14 weeks' gestation to 36 weeks) on the high-risk group by first-trimester combined screening reduces the risk of preterm-PE by $>60 \%[6,7]$. In contrast, the performance of first-trimester combined screening for term-PE is poor, with DR of $45 \%$ at FPR of $10 \%$ [2] and prophylactic use of aspirin is not beneficial [6, 7].

In this article we discuss available models of prediction for term PE.

\section{IMPORTANCE OF TERM PREECLAMPSIA}

One-third of all the cases of PE occur preterm and are associated with high levels of neonatal morbidity and mor- tality and severe maternal complications. Two-thirds of cases are term-PE (Fig. 1) [2].

Although term PE is traditionally considered less dangerous, mainly because delivery is safe at this stage for the fetus, it is not deprived of maternal complications and, because term PE is much more common than preterm PE, most of the maternal complications that will be diagnosed in association with PE will be actually confined to this group. In the Confidential Enquiry into Maternal Deaths form the United Kingdom, 50\% of all cases of maternal deaths attributable to PE were due to term PE [8]. The same point is made by a study from the United States where the authors reported 985 cases of severe maternal morbidity in association with PE developing after 34 weeks and only 289 with

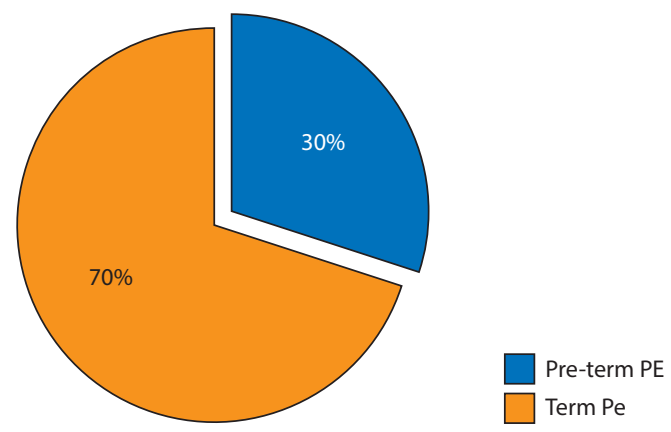

Figure 1. Incidence of preterm and term preeclampsia 
early-onset PE in a studied population of 670,120 singleton pregnancies [9]. Therefore, term-PE is not an innocuous condition; it should not be overlooked and prediction models and prevention strategies should be put forward.

\section{PATHOGENESIS OF TERM PREECLAMPSIA}

The development of PE requires both placental and maternal contributions (Fig. 2).

Preterm-PE in likely to reflect impaired placentation with failure of physiological transformation of the spiral arteries between 8 and 18 weeks of pregnancy. It is associated with fetal growth restriction, prominent placental pathology and abnormal Doppler velocimetry in the uterine arteries. By contrast, in term-PE the placental contribution seems less important, UTPI is often normal and there is usually normal fetal growth [10-13]. Rather, it seems that for term-PE, a maternal threshold for "tolerance" to the burden of pregnancy is achieved and that maternal characteristics such as being obese, having metabolic syndrome, comorbid conditions or insulin resistance are more likely to play a role [14-17].

Preterm- and term-PE, also appear to develop from different hemodynamic states. Term-PE appears to be more frequent in patients with high body mass index, increased cardiac output and relatively unchanged total vascular resistance; whereas patients with preterm-PE have lower BMI and relatively increased vascular resistance [18]

The angiogenic marker profile also seems to be different in preterm- and term-PE. Both are associated with altered serum levels of the anti-angiogenic marker soluble fms-like tyrosine kinase-1 (SFLT-1) and the pro-angiogenic PLGF, however the alterations are more pronounced in pretermthan term-PE [19]. Interestingly, for suspected PE before 35 weeks, a decrease in the maternal serum levels of PLGF has been shown to rule in women requiring delivery within

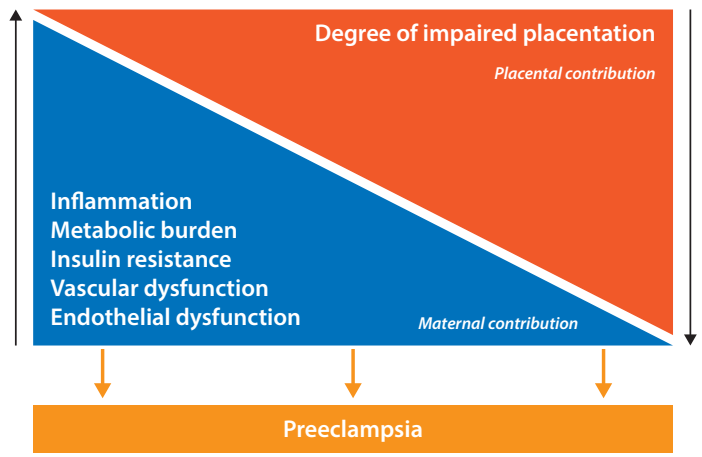

Figure 2. Preeclampsia requires both placental and maternal contributions for its development. An important degree of placental impairment would lead to manifestations of PE even in the absence of significant maternal contribution, while, in a mother with severe metabolic and vascular derangements, as with chronic hypertension, PE could develop with minimal placental contribution
14 days. However, this test's performance falls off in women presenting after 35 weeks [20,21].

\section{PREDICTION OF PREECLAMPSIA DEVELOPING AT TERM}

The traditional approach to screening for PE is to use a risk-scoring system based on maternal demographic characteristics and medical history (maternal factors) [22]. However, the performance of such approach, which essentially treats each risk factor as a separate screening test with additive detection rate and screen positive rate, is poor [23].

Another approach in screening for PE has been proposed by the Fetal Medicine Foundation (FMF) $[2,3]$ This approach, which is based on a survival time model, assumes that if the pregnancy was to continue indefinitely, all women would develop PE and whether they do so or not before a specified gestational age depends on a competition between delivery before or after development of PE. The effect of variables from maternal characteristics and history (maternal factors) and biomarkers is to modify the mean of the distribution of gestational age at delivery with $\mathrm{PE}$, so that in pregnancies at low risk for $\mathrm{PE}$ the gestational age distribution is shifted to the right with the implication that in most pregnancies delivery will occur before the development of PE. In high-risk pregnancies the distribution is shifted to the left. An algorithm combining maternal risk factors such as age, weight, racial origin, obstetric history, family history of $\mathrm{PE}$, method of conception, comorbidities as chronic hypertension, diabetes mellitus, systemic lupus erythematosus, antiphospholipid syndrome and MAP, UTPI, serum PLGF and SFLT-1 is used at different stages in pregnancy to detect those at high risk for $\mathrm{PE}$ and offer various interventions.

The performance of screening for term-PE by a combination of maternal factors with biomarkers (MAP, UTPI, PLGF and SFLT) at 12, 22 or 32 weeks' gestation is relatively poor with respective DR of about $45 \%, 45 \%$ and $65 \%$, at FPR of $10 \%[2,24,25]$. The best performance of screening for term-PE is achieved when screening is performed at 35-37 weeks, with DR of about $85 \%$ at FPR of $10 \%$ (Fig. 3) [26]. The values of MAP, UTPI and SFLT-1 are increased and serum PLGF is decreased compared to unaffected pregnancies. For all biomarkers the deviation from normal is inversely related to the gestational age at which delivery becomes necessary for maternal or fetal indications. We have therefore proposed that all women, irrespective of whether they had prior screening or not, should have assessment of risk at 35-37 weeks [27].

On the basis of the results from screening at 35-37 weeks the pregnancies can be stratified into three different management pathways (Fig. 4) [27]. 


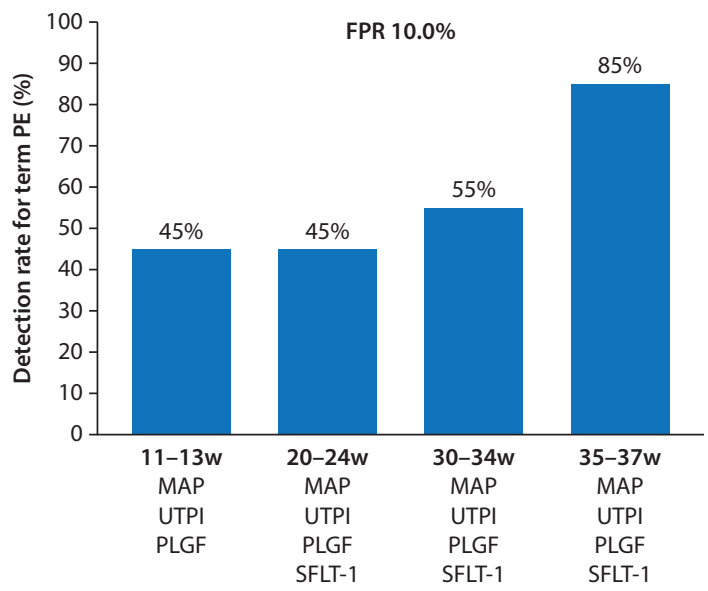

Figure 3. Detection rates in screening for term PE at 11-13, 20-24, 30-34 and 35-37 weeks using the FMF algorithm that combines materna factors and characteristics with mean arterial pressure (MAP), doppler assessment of the uterine artery pulsatility index (UTPI), placental growth factor (PLGF) and soluble Fms-like tyrosine kinese-1 (SFLT-1) FPR — false positive rate

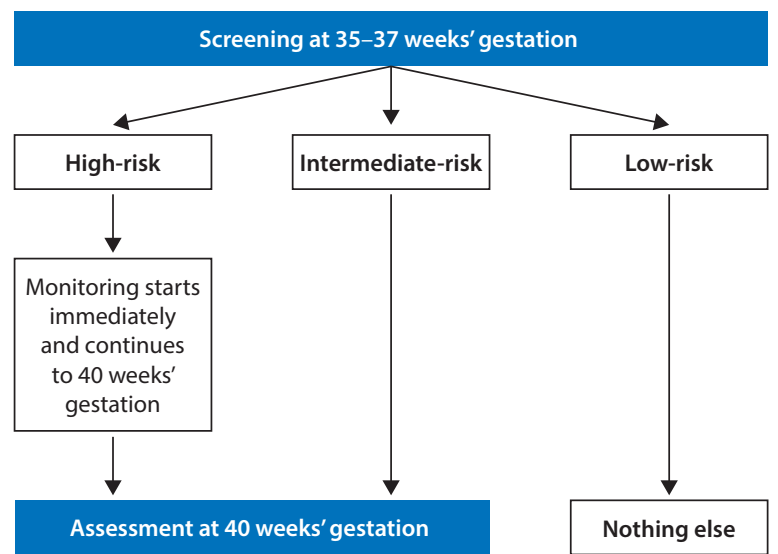

Figure 4. Stratification of pregnancies into high-, intermediateand low-risk management groups based on the estimated risk for preeclampsia at 35-37 weeks' gestation. The high-risk group would require intensive monitoring from the time of the initial assessment and up to 40 weeks' gestation, the intermediate-risk group would require reassessment at 40 weeks' gestation and the low-risk group would be managed expectantly

First, a low-risk group (risk for PE of $<1$ in 200) constitutes about $40 \%$ of the total and has a $>99.9 \%$ chance of not developing PE; this group can be reassured that development of PE is very unlikely and in the absence of any abnormal ultrasound findings or other obstetric indications the pregnancies can be managed expectantly awaiting for spontaneous onset of labor.

Second, a high-risk group (risk for PE at $<40$ weeks of $\geq 1$ in 100) constitutes about $20 \%$ of the total and contains $>90 \%$ of those that develop PE at $<40$ weeks. This group can be monitored by measurement of blood pressure and urinalysis at least on a weekly basis and the women can be advised to report any of the symptoms associated with severe $P E$, such as visual disturbance and epigastric pain. An alternative strategy that is currently being investigated by a major multicenter randomized study is to treat these pregnancies by pravastatin with the aim of reducing the risk of developing PE.

Third, an intermediate-risk group (risk between the high- and low-risk groups above). This group, together with the high-risk group that remains undelivered by 40 weeks' gestation, constitutes about $60 \%$ of the total and contains almost all cases of $\mathrm{PE}$ at $>40$ weeks. These pregnancies require reassessment at 40 weeks to decide the best time for delivery.

The cut-offs in risks to define the proportion of the population stratified into each of the three management groups and the protocols for such management will inevitably vary according to local preferences and health economic considerations. Future studies will examine whether the implementation of such protocols could improve perinatal outcome.

\section{CONCLUSIONS}

Term-PE is the most common form of PE and it can be associated with important maternal morbidity and mortality. The best performance of screening for term-PE is achieved by a combination of maternal factors, MAP, UTPI, PLGF and SFLT at 35-37 weeks' gestation. Using this approach, a DR of $85 \%$ can be achieved for FPR of $10 \%$. On the basis of the results from screening the pregnancies can be stratified into three different management pathways.

\section{Acknowledgments}

None.

\section{Conflict of interest}

None declared.

\section{REFFERENCES}

1. Say L, Chou D, Gemmill A, et al. Global causes of maternal death: a WHO systematic analysis. Lancet Glob Health. 2014; 2(6): e323-e333, doi: 10.1016/S2214-109X(14)70227-X, indexed in Pubmed: 25103301.

2. O'Gorman N, Wright D, Syngelaki A, et al. Competing risks model in screening for preeclampsia by maternal factors and biomarkers at 1113 weeks gestation. Am J Obstet Gynecol. 2016; 214(1): 103.e1-103. e12, doi: 10.1016/j.ajog.2015.08.034, indexed in Pubmed: 26297382.

3. O'Gorman N, Wright D, Syngelaki A, et al. A competing risks model in early screening for preeclampsia. Fetal Diagn Ther. 2012; 32(3): 171-178, doi: 10.1159/000338470, indexed in Pubmed: 22846473.

4. Akolekar R, Syngelaki A, Sarquis R, et al. Prediction of early, intermediate and late pre-eclampsia from maternal factors, biophysical and biochemical markers at 11-13 weeks. Prenat Diagn. 2011; 31(1): 66-74, doi: 10.1002/pd.2660, indexed in Pubmed: 21210481.

5. von Dadelszen P, Magee LA, Roberts JM. Subclassification of preeclampsia. Hypertens Pregnancy. 2003; 22(2): 143-148, doi: 10.1081/PRG 120021060, indexed in Pubmed: 12908998.

6. Rolnik DL, Wright D, Poon LC, et al. Aspirin versus Placebo in Pregnancies at High Risk for Preterm Preeclampsia. N Engl J Med. 2017; 377(7): 613-622, doi: 10.1056/NEJMoa1704559, indexed in Pubmed: 28657417.

7. Roberge S, Bujold E, Nicolaides $\mathrm{KH}$. Aspirin for the prevention of preterm and term preeclampsia: systematic review and metaanalysis. Am J Obstet Gynecol 2017; pii: S0002. 9378; 17: 32326-8, doi: 10.1016/j. ajog.2017.11.561. 
8. Cantwell R, Clutton-Brock T, Cooper G, et al. Saving Mothers' Lives: Reviewing maternal deaths to make motherhood safer: 2006-2008. The Eighth Report of the Confidential Enquiries into Maternal Deaths in the United Kingdom. BJOG. 2011; 118 Suppl 1: 1-203, doi: 10.1111/j.1471-0528.2010.02847.x, indexed in Pubmed: 21356004.

9. Lisonkova S, Sabr Y, Mayer C, et al. Maternal morbidity associated with early-onset and late-onset preeclampsia. Obstet Gynecol. 2014; 124(4): 771-781, doi: 10.1097/AOG.0000000000000472, indexed in Pubmed: 25198279.

10. Raymond D, Peterson E. A critical review of early-onset and late-onset preeclampsia. Obstet Gynecol Surv. 2011; 66(8): 497-506, doi: 10.1097/OGX.0b013e3182331028, indexed in Pubmed: 22018452.

11. Orabona R, Donzelli CM, Falchetti M, et al. Placental histological patterns and uterine artery Doppler velocimetry in pregnancies complicated by early or late pre-eclampsia. Ultrasound Obstet Gynecol. 2016; 47(5): 580-585, doi: 10.1002/uog.15799, indexed in Pubmed: 26511592.

12. Redman C. Pre-eclampsia: A complex and variable disease. Pregnancy Hypertension. 2014; 4(3): 241-242, doi: 10.1016/j.preghy.2014.04.009.

13. Hung $\mathrm{TH}, \mathrm{H}$ sieh TT, Chen SF. Risk of abnormal fetal growth in women with early-and late-onset preeclampsia. Pregnancy Hypertens. 2017 [Epub ahead of print], doi: 10.1016/j.preghy.2017.09.003, indexed in Pubmed: 29104027.

14. Bahado-Singh RO, Syngelaki A, Mandal R, et al. Metabolomic determination of pathogenesis of late-onset preeclampsia. J Matern Fetal Neonatal Med. 2017; 30(6): 658-664, doi: 10.1080/14767058.2016.118 5411, indexed in Pubmed: 27569705.

15. Erez $\mathrm{O}$, Romero R, Maymon $\mathrm{E}$, et al. The prediction of late-onset preeclampsia: Results from a longitudinal proteomics study. PLoS One. 2017; 12(7): e0181468, doi: 10.1371/journal.pone.0181468, indexed in Pubmed: 28738067.

16. Kwiatkowski S, Dołęgowska B, Kwiatkowska E, et al. Do the physiological aging of the placenta and the changes in angiogenesis marker sFlt-1 and PIGF concentrations predispose patients to late-onset preeclampsia? J Matern Fetal Neonatal Med. 2017 [Epub ahead of print]: 1-10, doi: 10. 1080/14767058.2017.1369517, indexed in Pubmed: 28816071.

17. Litwińska E, Litwińska $M$, Oszukowski $P$, et al. Biochemical markers in screening for preeclampsia and intrauterine growth restriction. Ginekol Pol. 2015; 86(8): 611-615, indexed in Pubmed: 26492710.
18. Vaddamani S, Keepanasseril A, Pillai AA, et al. Maternal cardiovascular dysfunction in women with early onset preeclampsia and late onset preeclampsia: A cross-sectional study. Pregnancy Hypertens. 2017; 10: 247250, doi: 10.1016/j.preghy.2017.10.010, indexed in Pubmed: 29111423.

19. Wikström AK, Larsson A, Eriksson UJ, et al. Placental growth factor and soluble FMS-like tyrosine kinase- 1 in early-onset and late-onset preeclampsia. Obstet Gynecol. 2007; 109(6): 1368-1374, doi: 10.1097/01. AOG.0000264552.85436.a1, indexed in Pubmed: 17540809.

20. Redman C. Diagnostic and predictive accuracy of placental growth factor in suspected pre-eclampsia. Pregnancy Hypertens. 2014; 4(3): 241, doi: 10.1016/j.preghy.2014.04.008, indexed in Pubmed: 26104637.

21. National Institute for Health and Care Excellence (2016). Diagnostic Guideline number 23. PIGF-based testing to help diagnose suspected pre-eclampsia.

22. National Institute for Health and Clinical Excellence (2010). Hypertension in pregnanacy.

23. O'Gorman N, Wright D, Poon LC, et al. Multicenter screening for pre-eclampsia by maternal factors and biomarkers at 11-13 weeks' gestation: comparison with NICE guidelines and ACOG recommendations. Ultrasound Obstet Gynecol. 2017; 49(6): 756-760, doi: 10.1002/uog.17455, indexed in Pubmed: 28295782.

24. Gallo DM, Wright D, Casanova C, et al. Competing risks model in screening for preeclampsia by maternal factors and biomarkers at 19-24 weeks' gestation. Am J Obstet Gynecol. 2016; 214(5): 619.e1-619.e17, doi: 10.1016/j.ajog.2015.11.016, indexed in Pubmed: 26627730.

25. Tsiakkas A, Saiid Y, Wright A, et al. Competing risks model in screening for preeclampsia by maternal factors and biomarkers at 30-34 weeks' gestation. Am J Obstet Gynecol. 2016; 215(1): 87.e1-87.e17, doi: 10.1016/j. ajog.2016.02.016, indexed in Pubmed: 26875953.

26. Andrietti $S$, Silva $M$, Wright $A$, et al. Competing-risks model in screening for pre-eclampsia by maternal factors and biomarkers at 35-37 weeks' gestation. Ultrasound Obstet Gynecol. 2016; 48(1): 72-79, doi: 10.1002/uog.15812, indexed in Pubmed: 26566592.

27. Panaitescu AM, Wright $D$, Militello $A$, et al. Proposed clinical management of pregnancies after combined screening for pre-eclampsia at 35-37 weeks' gestation. Ultrasound Obstet Gynecol. 2017; 50(3): 383-387, doi: 10.1002/uog.17419, indexed in Pubmed: 28133834. 